\title{
A European epidemiological survey of Vibrio splendidus clade shows unexplored diversity and massive exchange of virulence factors
}

\author{
Nasfi H. ${ }^{1}$, Travers Marie-Agnes ${ }^{2}$, De Lorgeril Julien ${ }^{3}$, Habib C. ${ }^{4}$, Sannie T. ${ }^{1}$, Sorieul L. ${ }^{1}$, Gerard J. ${ }^{1}$, \\ Avarre J. C. ${ }^{5}$, Haffner Philippe ${ }^{2}$, Tourbiez Delphine ${ }^{2}$, Renault Tristan ${ }^{2}$, Furones D. ${ }^{6}$, Roque A. ${ }^{6}$, \\ Pruzzo C. ${ }^{7}$, Cheslett D. ${ }^{8}$, Gdoura R. ${ }^{9}$, Vallaeys T. ${ }^{1, *}$
}

${ }^{1}$ CC 093 UMR 5119 "Ecologie des Systèmes Marins Côtiers", Université de Montpellier 2, place Eugène Bataillon, 34095, Montpellier, France

${ }^{2}$ Laboratoire de Génétique et Pathologie des Mollusque Marins, Institut Français de Recherche pour l'Exploitation de la Mer, av. du Mus de Loup, 17390, La Tremblade, France

${ }^{3}$ UMR 5119 "Ecologie des Systèmes Marins Côtiers", Institut Français de Recherche pour l'Exploitation de la Mer, pl E. Bataillon, 34095, Montpellier, France

${ }^{4}$ UR1077, Mathématique Informatique et Génome, Institut National de la Recherche Agronomique, Domaine de Vilvert, 78350, Jouy-en-Josas, France

${ }^{5}$ UMR 226, ISEM, Institut Recherche pour le Développement, pl E. Bataillon, 34095, Montpellier, France

${ }^{6}$ IRTA-Sant Carles de la Ràpita, Crta. Poble Nou, km. 5, 5, 43540, Tarragona, Spain 7. Department of Life, Earth and Environmental Sciences (DISTAV), University of Genoa, Genoa, Italy

${ }^{7}$ Department of Life, Earth and Environmental Sciences (DISTAV), University of Genoa, Genoa, Italy

${ }^{8}$ Fish Health Unit, Marine Institute, Rinville, Oranmore, Co., Galway, Ireland

${ }^{9}$ UR11ES70, Unité de recherche Toxicologie Microbiologie Environnementale et Santé, Faculté des sciences de Sfax, Université de Sfax, B.P. 1171, 3000, Sfax, Tunisia

*Corresponding author : T. Vallaeys, email address : $\underline{\text { tvallaey@univ-montp2.fr }}$

\begin{abstract}
:
The Vibrio splendidus clade has previously been associated with epidemic outbreaks of various aquatic animals, as in the case of the cupped oyster, Crassostrea gigas. To investigate whether involved strains could present a clonal origin and to identify possible alternative background carriage animals or zooplankton, a large epidemiological survey was conducted on isolates of the splendidus clade. For this purpose, Vibrio strains were isolated from various samples including oysters, mussels, sediments, zooplankton, and sea water on the basis of a North/South gradient of the European sea water zone (Ireland, The Netherlands, France, Italy, and Spain). A total of 435 isolates were successfully associated to the $\mathrm{V}$. splendidus clade using real time polymerase chain reaction with $16 \mathrm{~S}$ specific primers and probes. A multiple-locus variable-number tandem-repeat analysis (VNTR) was conducted on all isolates based on a multiplex PCR-VNTR with a set of primer pairs designed from the V. tasmaniensis LGP32 genome. Preliminary validation of the primers on a set of collection strains from the V. splendidus clade confirmed that the former V. splendidus-related LGP32 and relative strains were related to V. tasmaniensis rather than to the type strain V. splendidus LMG 4042. The VNTR analysis
\end{abstract}


was then successfully conducted on 335 isolates which led to the characterization of 87 different profiles. Our results showed that (1) the high diversity of VNTR did not enlighten significant correlation between a specific pattern and the origin of collected samples. However, populations isolated from animal samples tend to differ from those of the background environment; (2) oyster mortality events could not be linked to the clonal proliferation of a particular VNTR type. However, few different patterns seemed successively associated with samples collected during peaks of oyster's mortality. (3) Finally, no correlation could be seen between specific VNTR patterns and sequence phylogeny of the virulence factors vsm and ompU that were detected among strains isolated during as well as outside mortality events. These results, combined with incongruence observed between the ompU and vsm phylogenetic trees, suggested both large diffusion of strains and massive lateral gene transfer within the $V$. splendidus clade.

Keywords : VNTR, Vibrio splendidus, Epidemiology, vsm, ompU, Crassotrea giga, Mortality

\section{Introduction}

The Vibrio genus includes species known as pathogenic to various animals. Among other, the so called Vibrio splendidus clade forms a group presenting a large diversity, as it includes the type species $V$. splendidus, V. chagasii, V. crassostreae, V. cyclitrophicus, V. fortis, V. gigantis, V. kanaloae, V. lentus, V. pelagius, V. pomeroyi, and V. tasmaniensis (Sawabe et al. 2007, 2013; Thompson 2003a, $\underline{b}$ ) but also recently described species such as $\mathrm{V}$. toranzoniae (Lasa et al. 2013), or the less consensual species $\mathrm{V}$. artabrorum and V. atlanticus (Diéguez et al. 2011), V. celticus (Beaz-Hidalgo et al. 2010) and V. hemicentroti (Kim et al. 2013). Members of the V. splendidus clade appear ubiquitous, while frequently reported as common colonizers of marine and estuarine surface waters, but also sediments (Vezzulli et al. 2014). Others isolates can be found as commensals on the surface or within the intestinal flora of marine animals. Alternatively, other strains were reported to be pathogenic. Several members of the V. splendidus clade, for instance, are causing significant losses in the aquaculture industry worldwide. V. splendidus has been described as affecting fish species such as the turbot Scophthalmus maximus (Gatesoupe et al. 1999) but also, associated with mortalities of different mollusk species such as the larvae or spat of the Scallop Pecten maximus (Nicolas et al. 1996), the Scallop Patinopecten yessoensis (Liu et al. 2013) or the cupped oyster Crassostrea gigas (Lacoste et al. 2001; Le Roux et al. 2002) and other oyster species worldwide including C. virginica (Elston and Leibovitz 1980) and C. angulata (Comps 1988). However, a large plasticity was underlined at genetic and phenotypical level within the V. splendidus species (Le Roux et al. 2002, 2004). Indeed, both virulent (Lipp et al. 2001) and avirulent (Saulnier et al. 2010) V. splendidus isolates were reported by the literature. In V. splendidusrelated LGP32, virulence factors were identified that include the invasion porine OmpU (Duperthuy et al. 2010), a metalloprotease Vsm (Binesse et al. 2008; Le Roux et al. 2007), and an invasive vesicular serine protease Vsp (Vanhove et al. 2014). However, correlation is lacking between phenotypical virulence patterns of $\mathrm{V}$. splendidus-related strains and their virulence genotype. Alternatively, several authors have underlined the possibility of misclassification of several isolates as members of the different V. splendidus-related species. Indeed, the V. splendidus clade constitutes a complex of phenotypically and genetically related species (Macian et al. 2001; Le Roux et al. 2004; Thompson et al. 2003; Thompson 2003b). Misclassification, in the clade, is commonly due to close 16S rDNA gene sequences (Le Roux et al. 2004), combined with high sequence similarity of other genetic markers such as gyrB genes as for V. pomeroyi, V. kanaloae, and V. tasmaniensis (Le Roux et al. 2004). Other methods were used that include Pulsed Field Gel Electrophoresis (PFGE) (Chen et al. 2012), MultiLocus Sequence typing (MLST) (Cooper and Feil 2004; Maiden et al. 1998; Urwin and Maiden 2003), Amplified Fragment Length Polymorphism (AFLP) (Vos et al. 1995), microarrays (Schena et al. 1995; Pang et al. 2007; Vora et al. 2005), whole genome sequence comparison (Goudenège et al. 2013) or MLVA, have all been used to investigate the phylogeny, pathogenicity, or seasonal variations of the human pathogenic V. cholerae (Lutthisungnoen et al. 2008; Olsen et al. 2009; Teh et al. 2010) V. parahemolyticus (Kimura et al. 2008), V. vulnificus (Broza et al. 2009; Shekar et al. 2008), or the prawn 
pathogen V. nigripulchritudo (Goudenège et al. 2013). However, up to now, no attempts have been conducted to type mollusks pathogens. Moreover, fast and cheap methods are required for massive screening and epidemiological approaches of environmental Vibrio pathogens at large, international scale, as they can provide information on genetic relatedness of strains, inform about source of infection and allow the detection of particularly virulent strains, in a context of wide commercial transfer of animals. The aim of this study was thus to develop a multiplex high-resolution typing system for traceback analyses of $\mathrm{V}$. splendidus infections, based on polymorphism in seven VNTR loci. In this study, we present a novel VNTR based tool that was used for the classification of 435 geographically diverse isolates of the $\mathrm{V}$. splendidus clade from various sample types on the basis of a North/South gradient of the European sea water zone. In order to investigate possible proliferation of clonal virulent strains of the $\mathrm{V}$. splendidus clade during oyster 
mortality events, strains were isolated from oysters collected both during and outside mortality outbreaks on a European scale. Alternative potential reservoirs of such strains were also sampled including mussels (Mytilus edulis and Mytilus galloprovincialis), sea water, sediments and zooplankton. Here, our results underline the diversity of strains from the Vibrio splendidus clade, suggesting that misclassification of strains from this clade is much more frequent that originally thought. Further, this work demonstrates that this pathogen definitely do not present a clonal distribution, but conversely, that different clones seem replace each other during different mortality outbreaks, probably favored by massive lateral transfer of virulence factors thus underlying the epidemiological risk of emergence of 116 virulent strains, not strictly related to $V$. tasmaniensis.

\section{Materials and Methods}

\subsection{Strains used in this study and media}

The collection of Vibrio strains from the LGPMM/Ifremer, La Tremblade was used for validation of our Variable Number of Tandem Repeats (VNTR) approach. Collection strains used are listed in table 1. Alternatively, a total collection of 435 strains were collected from March 2011 to January 2013, from different European countries: France $n=324$ (Marennes- Oleron; Thau lagoon and Brest); Italy $n=75$ (Goro lagoon,), Spain $n=18$ (Fangar-Bay and Sor River), Ireland $n=10$ (County Donegal; County Waterford and County Louth) and the Netherlands $n=8$ (Lake Grevelinge), in majority from France. French samples were collected from the Atlantic coast with, ranging from North to South, sampling sites at Brest, and the Marennes-Oleron Bassin, and finally, from the Thau lagoon in the Mediterranean Coast, All samples were collected at sites of mollusks production. At those sites, isolation of strains was carried out from various sample types including the cupped oysters (Crassotrea gigas), during and between mortality outbreaks, mussels (both Mytilus edulis and Mytilus galloprovincialis), seawater, sediments, and filtered zooplankton (Supplementary Table S1). All strains were isolated from Thiosulfate-citrate-bile salts-sucrose (TCBS) agar medium (Difco, France), after preliminary enrichment in Zobell liquid medium for $24 \mathrm{~h}$ at $20^{\circ} \mathrm{C}$. Collection strains were retrieved from bacterial stock culture stored at $-80^{\circ} \mathrm{C}$ kept in $15 \%(\mathrm{v} / \mathrm{v})$ glycerol in Marine Broth.

\subsection{DNA extraction}

A maximum of five different colonies per sample were collected with a wooden sterile toothpick and put in suspension into $200 \mu \mathrm{l}$ of molecular biology grade water. Total DNA was extracted by heating the samples ten minutes at $98^{\circ} \mathrm{C}$. After a quick cooling to $4^{\circ} \mathrm{C}, 5 \mu \mathrm{l}$ were immediately used for the assay.

\subsection{Real-time PCR assay conditions for $V$. splendidus clade}

The identification of the isolates was confirmed by a Taqman QPCR-based diagnostic test aiming to detect all V. splendidus-related strains (Saulnier D, Travers MA, unpublished data) (Domeneghetti et al., 2014). Primers and probes used are presented in table 2A. Real-time PCR assays were conducted on a MX3000 Thermocycler (Stratagene), using Brilliant QPCR Core Reagent Kit (Stratagene). Each reaction was run in triplicate in a final volume of $25 \mu$ containing DNA sample $(5 \mu \mathrm{l}), 5 \mathrm{mM} \mathrm{MgCl} 2,200$ $\mu \mathrm{M}$ of each dNTP, $300 \mathrm{nM}$ of each primer, and $200 \mathrm{nM}$ of oligonucleotidic probe and 1.25 units of hot start Sure Taq polymerase. The thermal cycles were performed with a two-step PCR protocol: 1 cycle at $95^{\circ} \mathrm{C}$ for $10 \mathrm{~min}$, followed by 40 cycles at $95^{\circ} \mathrm{C}$ for $15 \mathrm{~s}$ and $60^{\circ} \mathrm{C}$ for $90 \mathrm{~s}$. Fluorescence intensity was expressed in delta reporters (dR) after background subtraction. Threshold was set using amplification based algorithm from MX3000 software (Stratagene). A common positive control was added to all plates. Threshold Cycle $(\mathrm{Ct})$ value corresponded to the PCR cycle number at which an increase in reporter fluorescence above a baseline signal was first detected. Before concluding about the status of the tested samples regarding the presence of Vibrio DNA, NTC should not present any amplification and the positive controls shouldfulfill the following requirements:

i) Difference between duplicated values should not exceed $1610.5 \mathrm{Ct}$, ii) A tested sample is considered positive if its $\mathrm{Ct}$ value is below 37. Positive strains were further submitted to VNTR analysis. 
2.4 Variable Number Tandem Repeats (VNTRs) search and primers design

A search for potential VNTR candidates in the genome of $V$. tasmaniensis (formerly $V$. splendidusrelated) LGP32 (Le Roux et al., 2009), was carried out (http://minisatellites.u psud.fr/). Primers were designed for each VNTR based on a 500 bp sequence covering the flanking and tandem repeat regions retrieved from the database. Primer 3 software pDraw and Netprimer were used to design the primers. The specificity of the primers and VNTR polymorphisms were tested with standard PCR using DNA templates from different groups of type or non-type Vibrio strains collection of the LGPMM/Ifremer, in order to validate our VNTR tools. 19 primer pairs (A to T) were initially assessed (Table 2A). Primers that gave redundant information with collection strains were further removed from the analysis. Combinations of remaining pairs were further assessed to optimize discrimination between collection strain species of the $V$. splendidus clade.

\subsection{PCR amplification of bacterial VNTR DNA}

Each $25 \mu \mathrm{l} \mathrm{PCR}$ mixture contained $5 \mu \mathrm{l}$ of $5 \times \mathrm{PCR}$ buffer, $0.5 \mu \mathrm{l}$ each deoxyribonucleotide $(10 \mathrm{mM}), 3 \mu \mathrm{l}$ of $\mathrm{MgCl} 2(25 \mathrm{mM}), 0.5 \mu \mathrm{l}$ of each primer $(10 \mu \mathrm{M}), 0.125 \mu \mathrm{l}$ of flexiTaq DNA polymerase (Promega, France) and $1 \mu \mathrm{l}$ of DNA template. The thermo-cycling conditions consisted of an initial denaturation step at $94^{\circ} \mathrm{C}$ for $5 \mathrm{~min}$, followed by 30 amplification cycles at $94{ }^{\circ} \mathrm{C}$ for $30 \mathrm{~s}, 58^{\circ} \mathrm{C}$ for $45 \mathrm{~s}$, and $72{ }^{\circ} \mathrm{C}$ for $30 \mathrm{~s}$ and a final polymerization step at $72^{\circ} \mathrm{C}$ for $7 \mathrm{~min}$. PCR products were electrophoresed in a $2 \%$ $(\mathrm{w} / \mathrm{v})$ agarose gel and visualized by ethidium bromide staining. Each sample was being tested in duplicate. The analysis of VNTRs was performed by using Gel Doc program. V. tasmaniensis (formerly $V$. splendidus related) LGP32 strain was use as positive controls in all reactions.

\subsection{PCR amplification of factors of virulence ( $\mathrm{vsm}$ and ompU)}

Sequence VS-2494 encoding the outer membrane protein ompU gene and sequence VS-1267 encoding the extracellular metalloprotease vsm, (extracted from UniProtKB B7VJI7), both essentials for the virulence and pathogenesis of the $V$. tasmaniensis (formerly $V$. splendidus related) LGP32 in the oyster (Binesse et al., 2008) (Duperthuy et al., 2010) were used for the design of the the ompU and the vsm primer pairs. The obtained primers sequences were first subjected to basic local alignment search tool (BLAST) analysis (Altschul et al., 1990) in the National Center for Biotechnology Information (NCBI) databases using the $V$. tasmaniensis (formerly $V$. splendidus) LGP32 genome (Le Roux et al., 2009) as target to avoid false priming sites and in order to check the universality of our primer pairs in different $V$. splendidus genomes. Multiple sequence alignment of Vibrio strains for ompU (GenBank accession number: BA000031; HM042874; CP006718; CP000789; BA000037) and for vsm (GenBank accession number AB734038; AB080995; AB540651), were then carried out using ClustalW (Thompson et al., 2002). The sequences of the primers are presented in table $2 \mathrm{~A}$.

For amplification of $V$. tasmaniensis virulence factors, $5 \mu \mathrm{l}$ of $5 \times \mathrm{PCR}$ buffer, $1 \mu \mathrm{l}$ each deoxyribonucleotide $(10 \mathrm{mM}), 3 \mu \mathrm{l}$ of $\mathrm{MgCl} 2(25 \mathrm{mM}), 0.5 \mu \mathrm{l}$ of each primer $(10 \mu \mathrm{M}), 0.125 \mu \mathrm{l}$ of Taq DNA polymerase (Promegaflexi. France) and $1 \mu$ of DNA template were mixed by reaction tube. The thermo-cycling conditions consisted of an initial denaturation step at $94^{\circ} \mathrm{C}$ for $5 \mathrm{~min}$, followed by 35 amplification cycles at $94^{\circ} \mathrm{C}$ for $30 \mathrm{~s}, 56^{\circ} \mathrm{C}$ for $45 \mathrm{~s}$, and $72{ }^{\circ} \mathrm{C}$ for $1 \mathrm{~min}$ and a final polymerization step at $72^{\circ} \mathrm{C}$ for $7 \mathrm{~min}$. PCR products were electrophoresed in a 1.5\% (w/v) agarose gel and visualized by ethidium bromide staining. Positive controls consisted, as previously, of $V$. tasmaniensis (formerly V. splendidus-related) LGP32.

\subsection{Sequencing}

PCR products selected for sequencing were purified with HighPrepTM 209 PCR Kit based on paramagnetic bead technology to remove salts, primers, primer-dimers, dNTPs following the manufacturer's instruction (MagBio Genomics, Inc.), and were then sequenced with BigDye ${ }^{\circledR}$ Terminator v3.1 Cycle Sequencing kit (Applied Biosystems, USA) using the forward and reverse primers used for PCR amplification. After removing unincorporated terminators from the Sanger sequencing reactions with the HighPrepTM DTR reagent (MagBio Genomics, Inc.), the sequences were performed on an Applied Biosystems 3130x1 Genetic Analyzer (Life Technologies, USA). 
For data analysis, the R -APE (Paradis et al., 2004) package were used. For this purpose, results for each different VNTR-PCR fragment obtained were converted into binary data using code 1 or 0 according to presence or absence of the fragment/ amplification in each strain, respectively. Twodimensional hierarchical clustering was then performed on $335 \mathrm{~V}$. splendidus generated by PCRVNTR out of the 435 strains assessed (100 strains gave no signal). 87 distinct patterns could be discriminated based on presence/absence of distinct VNTR fragments. Dissimilarity matrix was then used as entry for Neighbor Joining (NJ), using Euclidean distance and the tree was then rooted with midpoint rooting. Furthermore, similar coding scheme was used to encode occurrence or lack of PCR amplification with ompU and vsm primers, and isolation during or outside oyster's mortality peaks. Correspondence Analysis (Benzecri, 1992) was then performed on PCR amplification data for the 335 strains (representing individuals in the analysis), while the 19 variables were represented by 16 VNTR fragments (present or absent in each pattern), two OmpU and Vsm virulence factors (that were detected or not), and one 231 variable representing isolation during or outside mortality. Burt table (Burt, 1950), was then generated, by applying double coding of the data on each of the 19 variables (occurrence of 16 distinct VNTR fragments, occurrence of ompU and vsm amplification, isolation during or between oyster's mortality outbreaks). Burt table was further used as entry for Factor Analysis, for which we used the FactorMine R statistical package (Lê et al., 2008). Geographical origin, sample type, and year of collection were used as supplementary, explanatory variables.

\subsection{Diversity analysis}

The discrimination power of the MLVA assay was established by firstly calculating the Simpson's (Simpson, 1949), Shannon (Shannon, 1948) and Eveness (Carlo et al., 1998) diversity index for individual and a combined set of VNTR markers using the BioNumerics software: Simpson (D) Shannon $\left(\mathrm{H}^{\prime}\right)$ and Eveness $(\mathrm{E})$ indexes were estimated from the number of strains falling into the different recorded patterns $\mathrm{Ni}$ (i ranging from 1 to 87 in our case) out of the total strain number assessed in our study $(\mathrm{N}=435)$. Simpson diversity index was calculated as below:

$$
D=\sum_{1=1}^{N} \mathrm{Ni}(\mathrm{Ni}-1) / N(\mathrm{~N}-1)
$$

Shannon Index was estimated as follows:

$$
H^{\prime}=-\sum_{\mathrm{i}=1}^{S} \mathrm{pi}(1 \mathrm{n} \mathrm{pi})
$$

Where $\mathrm{pi}=\mathrm{Ni} / \mathrm{N}$

Eveness Index was estimated as follows:

$E=H^{\prime} / H \max$

Where Hmax $=\log 2(\mathrm{~N})$

\subsection{Neighbor joining of ompU and vsm sequences}

For validation purposes and to check lack of false positive amplification, sequences were first subjected to basic local alignment search tool (BLAST) analysis (Altschul et al., 1990) in the National Center for Biotechnology Information (NCBI) databases using The $V$. tasmaniensis (formerly $V$. splendidus- related) LGP32 genome as target. Multiple sequence alignment was then carried out using ClustalW (Thompson et al., 2002) at the European Bioinformatics Institute Website (http://www.ebi.ac.uk/Tools/msa/clustalw2/). Phylogenetic analyses were then performed on obtained alignments, using MEGA5 (Tamura et al., 2007). Phylogenetic trees of both vsm and ompU sequences among positive isolates were constructed by the Neighbour-joining method (Nei \& Li, 1979). The robustness of the inferred tree was evaluated by bootstrapping (1000 replications). 


\subsection{VNTR validation}

A set of 19 loci carrying tandem repeats presented in variable number were deduced from the genome of the type V. tasmaniensis (formerly V. splendidus- related) LGP32 (Le Roux et al., 2009). Each primer pairs were selected for their ability to differentiate as far as possible isolates from the $V$. splendidus sensus stricto species, among which the VNTR primer B appeared specific to the LGP32 strain (Table 2 B). However, we failed to design a set of primers that could be specific for all previously identified $V$. splendidus sensus stricto species as signals were clearly different between the two $V$. tasmaniensis (formerly $V$. splendidus related) LGP32 and LGP31 and the type 273 strain $V$. splendidus LMG4042. Our primer sets were then validated on the type strain collection including strains of the $V$. splendidus clade $(V$. gigantis, $V$. crassostreae, $V$. cyclitrophicus, $V$. tasmaniensis, $V$. pomeroyi, $V$. lentus, $V$. kanaloae, $V$. pelagius and $V$.chagasii and $V$. fortis) as redefined recently by (Sawabe et al., 2013) which all showed a signal with at least one of the designed primer sets except for $V$. fortis, thus questioning the recent reclassification of $V$. fortis within the $V$. splendidus clade (Sawabe et al., 2013). More distant strains of Vibrio species (V. harveyi, V. tapetis, V. aestuarianus,), that were also assessed, gave no amplification signal when our primers were used, demonstrating there by the specificity of our tools for the $V$. splendidus clade (Table 2 B). Globally, for most primers tested, the two strains LGP32 and LGP31 showed signals related to those obtained with $V$. tasmaniensis and to a lesser extent to $V$. kanaloae, but not to the $V$. splendidus type strain LMG4042 (Table $2 \mathrm{~B}$ ). This confirmed that virulent isolates, for long time classified as $V$. splendidus, are related to the $V$. tasmaniensis species, as recently underlined by (Sawabe et al., 2013). By further eliminating redundant primers sets, we came out with a multiplex, 7 primer pair based PCR tool set namely $A, C$, $\mathrm{E}, \mathrm{F}, \mathrm{L}, \mathrm{O}$, and R (Table $2 \mathrm{~B}$ ), that successfully amplified all collection strains from the $\mathrm{V}$. splendidus clade (except $V$. fortis) while providing clearly distinct patterns for each species of the clade, and gave no signal with more distant strains of Vibrio species (V. harveyi, V. tapetis, V. aestuarianus,).

\subsection{VNTR analysis applied during epidemiological survey}

To investigate whether involved strains could present a clonal origin and to identify possible alternative background carriage animals or zooplankton, a large epidemiological survey was conducted on isolates of the $V$. splendidus clade.The multiplex PCR-VNTR approach was successfully applied on the strain collection isolated 296 during the 3 years field survey, from various samples including oysters (C. gigas), mussels ( $M$. edulis and $M$. galloprovincialis), sediments, zooplankton and sea water on the basis of a North/ South gradient of the European sea water zone (Ireland, The Netherlands, France, Italy, and Spain), A total of 435 isolates, were associated to the splendidus clade using real time PCR with dedicated $V$. splendidus clade $16 \mathrm{~S}$ specific primers. Strains geographical origin and sample type are presented in figure 1; (Supplementary Table S1). Most of strains were sampled in France (altogether 324 strains). Most of $V$. splendidus strains were isolated from $C$. gigas $(n=302), M$. edulis $(n=55)$, Zooplankton $(n=23)$, Seawater $(n=23)$, Sediment $(n=15)$ and M. galloprovincialis $(n=17)$ during different peaks of oyster mortality $(n=148)$ or between peaks $(n=116)$. Most of the strains were isolated during the first two years (2011: $n=117$ and 2012: $n=236$ ). The VNTR analysis was successfully conducted on 335 isolates out of the 435 assessed. Indeed, 100 isolates gave no amplification with our primer pairs suggesting that they could be more distantly related to the $V$. splendidus clade. Altogether, 87 distinct profiles were obtained (an illustration of the diversity of the patterns obtained is presented in figure 2). Globally, no clonal origin of $V$. splendidus could be demonstrated in this study. Conversely we observed a pan mixing scheme of the $V$. splendidus population. Indeed, neighbor-joining based tree reconstruction of VNTR data could discriminate 6 clusters (Fig. 3 a,b,c and d). A first cluster grouped strains related to the $V$. pelagius and $V$. chagasii species. This cluster gathered most of strains collected from France and Italy from different type of samples (sea water, mussels, oysters and zooplankton) and contained $26 \%$ of samples collected during oyster mortality (Fig. 3 a). A second cluster, included the $V$. tasmaniensis type strain and related isolates, including the strains LGP31 and LGP32, previously $V$. splendidus, now reclassified in the $V$. tasmaniensis species. This cluster grouped mainly French isolates collected from $C$. gigas and M. edulis in 2011-2012, but 320 was also noticed among Spanish and Italian isolates from different type of samples (sediment, mussels and sea water) showing large geographical dissemination from Atlantic up to Mediterranean waters. This second cluster contained $33 \%$ of samples collected during oyster mortality (Fig. 3, Supplementary Fig. 2A). A third cluster grouped $V$. lentus and $V$. splendidus LMG4042 type strains as well as $V$. cyclitrophicus and related isolates. Interestingly, this cluster gathered isolates from all countries, but grouped particularly all French isolates collected from oysters in 2011-2012. This cluster presents $62 \%$ of strains collected during oyster mortalities, and contains the 
dominant pattern (pattern 1) which was observed 44 times out of 335 isolates (and thus represented $13 \%$ of the strains assessed). This pattern was associated preferentially with oysters (31 occurrences in oysters out of 44 samples presenting this pattern i.e. $70.4 \%$ ) but could also be found among isolates issued from water column, M. edulis, sediments and zooplankton. Among strains presenting this pattern $66 \%$ were isolated during peaks of oyster mortality. This group, however, presented no relatedness with the virulent strains LGP32-LGP31 (Fig. 3, Supplementary Fig. 2B). A fourth cluster associated $V$. pomeroyi, $V$. crassostreae and $V$. gigantis as well as environmental isolates from all countries from years 2011 and 2012 and contained a weak number of samples collected during oyster mortality (35\%). This cluster included strains presenting the second dominant pattern (pattern 3) as it occurred 42 times (and thus represented $12.5 \%$ of the total strains assessed), preferentially collected out of peaks of oyster mortality. This pattern was preferentially associated with oysters (30 occurrences in oysters out of 42 samples presenting this pattern i.e. $71.4 \%$ ) but could also occasionally be found among isolates issued from water column, $M$. edulis and zooplankton. It was mostly associated with French samples but could also be found in Ireland and Italy (Fig. 3, Supplementary Fig. 2C). Finally, the five and six clusters included our isolates only, but no reference strains, 344 suggesting that further investigations should be performed on representatives of these groups. Cluster 6 presented higher proportion of strains collected during oyster mortalities, and included the third dominant pattern (pattern 5), preferentially associated with oysters (18 occurrences in oysters out of 21 samples presenting this pattern i.e. 85\%) (Fig. 3, Supplementary Fig. 2D). This pattern, like pattern 1 in cluster 3 , was preferentially isolated during mortality periods as 16 strains out of 21 presenting this pattern were isolated during mortality peaks. Finally, different VNTR patterns, for instance pattern number (43 and 26) could be found in a same sample (geographical origin, sample type, date of sampling and even animal sampled when a host was involved. Globally, a tendency to associate patterns 1 and 5 to oyster mortality events could be drawn from cluster analysis but, although related, no isolates shared strictly the same VNTR pattern as the highly virulent strain $V$. tasmaniensis LGP32.

\subsection{Virulence genes analysis during epidemiological survey}

In order to investigate whether dominant VNTR patterns were or not harboring virulence genes, and/or could be associated to mortality outbreaks, we sequenced two genes ompU and vsm involved in the pathogenesis of $V$. tasmaniensis LGP32. The list of accession number of ompU and $v s m$ are presented in supplementary table S2. Our results demonstrate that the ompU and vsm phylogenetic trees were incongruent (Fig. 4) and (Fig.5). The ompU phylogenetic tree divides strains into 3 branches that do not discriminate strains according to temperature, year of collection, mortality level or locations. The vsm phylogenetic tree neither divides strains into 4 branches that do not correlate with neither variable assessed (temperature, year of collection, mortality level or locations) nor groups resulting from the ompU phylogenetic analysis. A unique cluster (cluster 3 ) from the ompU tree (Fig. 4) gathers strains grouping also together in a same vsm 367 cluster (cluster 4) (Fig.5), but not in a same VNTR cluster. Moreover, strains from the VNTR cluster to which belongs $V$. tasmaniensis LGP32, do not share ompU or vsm sequence similarity and none of the ompU genes sequenced among our isolates showed identity with the $V$. tasmaniensis LGP32 ompU gene sequence (Fig4). Only one isolate ( $\mathrm{N}^{\circ} 207$ ) showed highly similar, although not strictly identical vsm sequence (Fig 5), but presented a VNTR pattern distantly related to the $V$. tasmaniensis LGP32 one (Fig 3). Interestingly, this strain showed a positive amplification with LGP32 specific VNTR primer pair B (data not shown). Finally, no correlation could be established between PCR based detection of virulence factors and specific location, date, mortality periods, or sample type. Further, the phylogenetic approach performed on all obtained sequences only showed close relationships between samples from the same date and location only, suggesting, in some cases, redundant sampling. This analysis thus underlines globally the incongruence of phylogenetic reconstructions performed with sequences of two virulence genes and could suggest independent horizontal gene transfer. Furthermore, both phylogenetic trees build with sequences of virulence genes are incongruent with cluster obtained from VNTR data.

\subsection{Diversity analysis}

Diversity analysis confirmed factor analysis as Shannon ( $\left.\mathrm{H}^{\prime}\right)$, Eveness (E) and Simpson (D) indexes were estimated as $H^{\prime}=1.586, E=0.628$ and $D=0.0413$, respectively, the last index taking into account both, the size of the population (sample size) and the distribution of the pattern types among the population assessed (peaked or evenly distributed). Although a significant diversity of patterns was 
observed in the assessed bacterial population, as suggested by both Simpson and Eveness indexes values, an average value of 0.04 for the Simpson index was obtained (Simpson index ranges from zero to one, with zero for the most diverse profiles and one for those less diversified) and an 391 average value of 0.63 was obtained for the Eveness index (evenness ranges from zero to one, with zero signifying no evenness and one, a complete evenness).

\subsection{Factor analysis}

Factorial correspondence analysis is indeed a multiparametric approach based on the Chi2 metric (Benzecri, 1992). Here, the first two factorial axis summed $19.26 \%$ of the total inertia of the data only, with an explained variability of $9.98 \%$ for axis 1 and $9.28 \%$ for axis 2 , respectively. The analysis, showed a tendency to split strains according to both their geographical origin, and sample type, as shown in supplementary Fig. 1A and Fig. 1B, respectively. Indeed, the first axis globally distinguished environmental strains (isolated from zooplankton, sediment or sea water) from strains isolated from animals (whatever host type, mussels or oyster) (Supplementary Fig. 1B). When geographical origin was used, however, as supplementary variable for projection, second axis distinguished similarly isolates according to country of origin, with a clear split between Italian samples, thus underlying a biased sampling (most environmental isolates were indeed originated from Italy) (Supplementary Fig. 1A). However all this suggest both a global split between Mediterranean and Atlantic populations of the $V$. splendidus clade and a tendency of preferential association of given genotypes with animals that should, indeed, be further investigated and confirmed with more orthogonal sampling. Finally, the variable mortality outbreak seems to be orthogonal to previous variables as it contributes most to axis two. Further, occurrence of $v s m$ and ompU amplification correlated globally together but could not be associated to isolation during mortality outbreaks (Supplementary Fig. 1C). Conversely, isolation during mortality outbreak could preferentially be associated with occurrence of pattern 1 and 5 in VNTR amplification (Supplementary Fig. 1C), confirming results from cluster analysis (Fig. 3).

\section{Discussion}

Different strains related to the Vibrio splendidus clade have previously been associated with epidemic outbreaks of various aquatic animals, especially, marine bivalves (Lacoste et al., 2001) (Gómez-León et al., 2005) (Liu et al., 2013). V splendidus was also reported to be associated to mass mortality outbreaks of Crassostrea gigas (Waechter, Le Roux, Nicolas, Marissal, \& Berthe, 2002)(Gay et al., 2004). To investigate whether or not such strains could present a clonal origin, but also to identify possible alternative background carriage animals, a large scale epidemiological survey was conducted on a pan European scale on isolates of the $V$. splendidus clade. However, due to the difficulty to discriminate between interspecific and intraspecific diversity of the $V$. splendidus species and clade respectively, and to infer epidemiological relationships between occurrence of given strains and epidemiological outbreaks, it was clearly stated that typing methods used up to know were not fully appropriate (Le Roux et al., 2004) (Sawabe et al., 2007) (Thompson et al., 2005). For this purpose, a Variable Number Tandem Repeat (VNTR) based multiplex PCR was set up allowing simultaneously a fast detection and identification of these species and further typing. Recently, VNTR has become a widely used method in epidemiology as it allows high throughput, discriminatory and reproducible analysis of large amounts of isolates and wide scale inter-laboratory comparisons (Stine, Alam, Tang, Sack, \& Morris, 2008) (Kattar et al., 2008) (Lindstedt, 2005). However, up to now, its use has mainly been restricted to bacterial species of clinical importance. Here, we show that it can be accurately applied to the typing of environmental isolates while providing a reasonable discrimination rate compared to other techniques such as MLST which appeared much discriminative and unable to provide information about strain migration, transfer or epidemiological outbreak in a previous study (Thompson et al., 2005). By using MLST in a previous large scale investigation, these authors came out with over 1000 strain types, failing to deduce 439 any relationships between so diverse information. However, such investigation could have been more informative when performed on a specific sample type and, or restricted to a specific sub-clade of the Vibrio genus. Here we first show that large scale epidemiological investigations could be applied to environmental situations at large scale and escape the strict domain of epidemiology of human pathogens.

Our first results, obtained while validating our method on collection strains was that LGP31 and LGP32 strains could belong to the $V$. tasmaniensis rather than to the $V$. splendidus species (Fig. 3 ), confirming the recent reclassification of these strains in the $V$. tasmaniensis species (Sawabe et al., 2013). Misclassification and to some extend over classification of strains of the V.splendidus clade has indeed led, for long, to false conclusions. Our tool should help further systematic typing. However, 
no strictly significant conclusions could be drawn from our data on strict association of these species with given habitat, as related isolates could also be found in alternative sample types including zooplankton and seawater, thus confirming results of (Hunt et al. 2008) and (Preheim et al. 2011). These data also confirm that species from the $V$. splendidus clade, as other Vibrio, can present alternative hosts (Liu et al., 2013). Further investigations are needed among more diverse animal samples to define more precisely alternative host's species for the $V$ splendidus clade, if they exist. Further, a wide diversity of VNTR patterns was obtained during our study, far exceeding our expectations and preliminary data obtained from collection strains suggested that the specific diversity of the clade is not extensively investigated and that novel species may exist, especially among environmental isolates. This, also can be linked to the previously reported genomic plasticity within the V. splendidus clade (Le Roux et al., 2009) (Thompson et al., 2005), still suggesting rapid evolution, either by massive horizontal gene transfer events, or other mechanisms, like competence, as related genes seems complete 463 in the published $V$. tasmasniensis LGP32. In our case, although a large diversity of strains could be found, it appeared reduced when compared to these previous observations. However, diversity at local scale was confirmed by our results as different VNTR patterns could be found in a same sample, which is globally in accordance with a dissemination of strains associated with current practices of massive transfers of animals at pan European scale, especially for oysters (Meistertzheim et al., 2013). Although the large diversity observed, our study suggests that related strains from the $V$. splendidus clade from the VNTR group's 1 and 5 , rather than strictly clonal populations could be associated to mortality events, that could result from the dissemination and propagation of particular clones. However, our data draw only tendencies, despite of the number of strains already analysed. Much more strains have to be further analyzed, especially from environmental origin. However, our results confirm that diffusion of strains is particularly extended, at a European scale. Although a gradient can be seen between $V$. splendidus populations from Atlantic and Mediterranean coasts, identical types can also be observed. This could be related to marine streams but also typically to nursing practices. Extreme care should be taken by professionals before moving animals, especially in epidemic circumstances.

Further, the extended diversity observed within the $V$ splendidus clade did not allow us to clearly discriminate virulent from non-virulent strains. This led us to further assess the occurrence of two major virulence genes among our population, namely vsm and ompU (Binesse et al., 2008) (Duperthuy et al., 2010). However, as reported previously by Le Roux, these virulence factor were also harbored by environmental strains, as it was the case for the two V. splendidus 12BO1 and Med222 isolates (Le Roux et al., 2009).

The OmpU porin has indeed been shown to constitute 486 a major virulence factor among Vibrio strains and particularly among $V$. splendidus related species (Duperthuy et al., 2010). Indeed, it plays an active role in host pathogen interactions, serving as adhesion protein (Goo et al., 2006), while also being involved in the resistance to antimicrobial peptides (Mathur \& Waldor, 2004) (Mathur et al., 2007). However, this porin seems maintained in environmental strains. A metalloprotease Vsm, found to constitute the main toxic factor of secreted extracellular products (Binesse et al., 2008) (Le Roux et al., 2007) was also present in environmental strains. Interestingly the sequence diversity of these two genes among our strains was larger than the one previously reported by Liu et al (2013) for strains isolated from the Yesso scallop (Patinopecten yessoensis) (Liu et al., 2013) and no correlation could be noticed between ompU and vsm phylogenies suggesting that the two assessed virulence genes could be recruited and, or lost, independently. Similarly, no clear association could be find between occurrence of ompU, vsm and given VNTR patterns suggesting potential horizontal transfer of this virulence factor among isolates. Transfer of these key factors of pathogenicity between strains has to be further taken care of as support of emergence of virulent phenotypes. From genome data, there is strictly no evidence of mobility genes associated to neither ompU nor vsm virulence factors. Indeed, ompU (VS-2494) is adjacent on the chromosome 1 to house-keeping genes including a D-alanyl-Dalanine carboxypeptidase on one side, and, on the other side, the transcription elongation factor GreA, as well as a predicted RNA-binding protein (Le Roux et al., 2009). Similar observations can be deduced from the genomic sequence of chromosome 1 of $V$. splendidus (accession number FM954972, (Le Roux et al., 2009) for vsm (VS-1267) although for this gene, published annotation mentions mainly conserved genes of unknown function as genomic neighbours. Consequently, we lack interpretation of mechanisms underlying genetic exchanges in these areas of the $V$. splendidus genome 510 and one should further focus in the comparative organisation of genomic sequences of $V$. splendidus strains in these area. However our data are not sufficient to conclude if this exchange is or not restricted to virulence factors and pan3 genome approach of the clade should be over taken that only could delineate its extend. Unfortunately, genome data of all strains of the clade are not yet available. This large scale study clearly underlined the genetic diversity of the $V$. splendidus clade and 
its potential genomic plasticity. Our results enlighten particularly the fact that this clade harbours potentially not yet described species. Our VNTR approach was thus showed to constitute a powerful, rapid and inexpensive tool for direct, one-step screening and simultaneous typing of $V$. splendidus and related species but robustness of VNTR grouping in link with taxonomy has not been yet clearly assessed. However it clearly appears as an in valuable tool for wide scale preliminary investigations of $V$. splendidus clade diversity. Finally, this study clearly underlines the massive dissemination of strains from the Vibrio splendidus clade throughout Europe as no sign of clonality could be detected even at a local scale. This can result from diffusion through marine streams, and contribution of aquaculture practices could not be excluded.

\section{Acknowledgments}

This study has been carried out in part with financial support from the Commission of the European Communities, under the EC's Seventh Framework Program (FP7/2007-2013) in the grant funded "KBBE.2010.1.2-08: Improving European mollusc aquaculture: disease detection and management". It does not necessarily reflect its views and in no way anticipates the Commission's future policy in this area.

We also aknowledge the EMIDA project 532 MOLTRAQ for financial support, Egide for funding the grant number 18470SA, the CMCU for grant number 08G0908, and AUF for granting the second PhD year from Hanen Nasfi. Finally, the data used in this work were partly produced through the technical facilities of the Centre Mediterranean Environment Biodiversity, University of Montpellier 2, France. Finally, special thanks to Mr Marc Englezma for providing us with isolates from Netherlands.

\section{References}

Altschul, S. F., Gish, W., Miller, W., Myers, E. W., \& Lipman, D. J. (1990). Basic local alignment search tool. Journal of Molecular Biology, 215(3), 403-10. doi:10.1016/S0022-2836(05)80360-2

Beaz-Hidalgo, R., Diéguez, A. L., Cleenwerck, I., Balboa, S., Doce, A., de Vos, P., \& Romalde, J. L. (2010). Vibrio celticus sp. nov., a new Vibrio species belonging to the Splendidus clade with pathogenic potential for clams. Systematic and Applied Microbiology, 33(6), 311-5. doi:10.1016/j.syapm.2010.06.007

Benzecri, J.-P. (1992). Correspondence Analysis Handbook (p. 688). New York: Marcel Dekker.

Binesse, J., Delsert, C., Saulnier, D., Champomier-Vergès, M.-C., Zagorec, M., Munier-Lehmann, H. Le Roux, F. (2008). Metalloprotease vsm is the major determinant of toxicity for extracellular products of Vibrio splendidus. Applied and Environmental Microbiology, 74(23), 7108-17. doi:10.1128/AEM.01261-08

Broza, Y. Y., Danin-Poleg, Y., Lerner, L., Valinsky, 552 L., Broza, M., \& Kashi, Y. (2009). Epidemiologic study of Vibrio vulnificus infections by using variable number tandem repeats. Emerging Infectious Diseases, 15(8), 1282-5. doi:10.3201/eid1508.080839

Burt, C. (1950). The factorial analysis of qualitative data. British Journal of Statistical Psychology, 3(3), 166-185.

Carlo H.R. Heip, P. M. J. H. \& K. S. (1998). Indices of diversity and evenness *. Océanis, 24 (4 ), 6187.

Chen, M., Li, L.-P., Wang, R., Liang, W.-W., Huang, Y., Li, J., ... Gan, X. (2012). PCR detection and PFGE genotype analyses of streptococcal clinical isolates from tilapia in China. Veterinary Microbiology, 159(3-4), 526-30. doi:10.1016/j.vetmic.2012.04.035

Comps, M. (1988). Epizootie Diseases of Oysters Associated with Viral Infections. American Fisheries Society, 18(Elston 1979), 23-37.

Cooper, J. E., \& Feil, E. J. (2004). Multilocus sequence typing--what is resolved? Trends in Microbiology, 12(8), 373-7. doi:10.1016/j.tim.2004.06.003

Diéguez, A. L., Beaz-Hidalgo, R., Cleenwerck, I., Balboa, S., de Vos, P., \& Romalde, J. L. (2011). Vibrio atlanticus sp. nov. and Vibrio artabrorum sp. nov., isolated from the clams Ruditapes philippinarum and Ruditapes decussatus. International Journal of Systematic and Evolutionary Microbiology, 61(Pt 10), 2406-11. doi:10.1099/ijs.0.025320-0

Domeneghetti, S., Varotto, L., Civettini, M., Rosani, U., Stauder, M., Pretto, T., Venier, P. (2014). Mortality occurrence and pathogen detection in Crassostrea gigas and Mytilus galloprovincialis closegrowing in shallow waters (Goro 572 lagoon, Italy). Fish \& Shellfish Immunology. doi:10.1016/j.fsi.2014.05.023

Duperthuy, M., Binesse, J., Le Roux, F., Romestand, B., Caro, A., Got, P., ... Destoumieux-Garzón, D. (2010). The major outer membrane protein OmpU of Vibrio splendidus contributes to host antimicrobial peptide resistance and is required for virulence in the oyster Crassostrea gigas. Environmental Microbiology, 12(4), 951-63. doi:10.1111/j.1462-2920.2009.02138.x 
Elston, R., \& Leibovitz, L. (1980). Pathogenesis of Experimental Vibriosis in Larval American Oysters, Crassostrea virginica. Canadian Journal of Fisheries and Aquatic Sciences, 37(6), 964-978. doi:10.1139/f80-126

Gatesoupe, F. J., Lambert, C., \& Nicolas, J. L. (1999). Pathogenicity of Vibrio splendidus strains associated with turbot larvae, Scophthalmus maximus. Journal of Applied Microbiology, 87(5), 757763. doi:10.1046/j.1365-2672.1999.00922.x

Gay, M., Renault, T., Pons, A.-M., \& Le Roux, F. (2004). Two vibrio splendidus related strains collaborate to kill Crassostrea gigas: taxonomy and host alterations. Diseases of Aquatic Organisms, 62(1-2), 65-74. doi:10.3354/dao062065

Gómez-León, J., Villamil, L., Lemos, M. L., Novoa, B., \& Figueras, A. (2005). Isolation of Vibrio alginolyticus and Vibrio splendidus from aquacultured carpet shell clam (Ruditapes decussatus) larvae associated with mass mortalities. Applied and Environmental Microbiology, 71(1), 98-104. doi:10.1128/AEM.71.1.98-104.2005

Goo, S. Y., Lee, H.-J., Kim, W. H., Han, K.-L., Park, D.-K., Lee, 592 H.-J., ... Park, S.-J. (2006). Identification of OmpU of Vibrio vulnificus as a fibronectin-binding protein and its role in bacterial pathogenesis. Infection and Immunity, 74(10), 5586-94. doi:10.1128/IAI.00171-06

Goudenège, D., Labreuche, Y., Krin, E., Ansquer, D., Mangenot, S., Calteau, A., ... Le Roux, F. (2013). Comparative genomics of pathogenic lineages of Vibrio nigripulchritudo identifies virulenceassociated traits. The ISME Journal, 7(10), 1985-96. doi:10.1038/ismej.2013.90

Kattar, M. M., Jaafar, R. F., Araj, G. F., Le Flèche, P., Matar, G. M., Abi Rached, R., Vergnaud, G. (2008). Evaluation of a multilocus variable-number tandem-repeat analysisscheme for typing human Brucella isolates in a region of brucellosis endemicity. Journal of Clinical Microbiology, 46(12), 393540. doi:10.1128/JCM.00464-08

Kim, D., Baik, K. S., Hwang, Y. S., Choi, J. S., Kwon, J., \& Seong, C. N. (2013). Vibrio hemicentroti sp. nov., an alginate lyase producing bacterium, isolated from the gut microflora of sea urchin (Hemicentrotus pulcherrimus). Int J Syst Evol Microbiol. doi:10.1099/ijs.0.047951-0

Kimura, B., Sekine, Y., Takahashi, H., Tanaka, Y., Obata, H., Kai, A., ... Fujii, T. (2008). Multiplelocus variable-number of tandem-repeats analysis distinguishes Vibrio parahaemolyticus pandemic O3:K6 strains. Journal of Microbiological Methods, 72(3), 313-20. doi:10.1016/j.mimet.2007.12.014

Lacoste, A., Jalabert, F., Malham, S., Cueff, A., Gélébart, F., Cordevant, C., Poulet, S. (2001). A Vibrio splendidus strain is associated with summer mortality of juvenile oysters Crassostrea gigas in the Bay of 614 Morlaix (North Brittany, France). Diseases of Aquatic Organisms, 46(2), 139-45. doi:10.3354/dao046139

Lasa, A., Diéguez, A. L., \& Romalde, J. L. (2013). Vibrio toranzoniae sp. nov., a new member of the Splendidus clade in the genus Vibrio. Systematic and Applied Microbiology, 36(2), 96-100. doi:10.1016/j.syapm.2012.11.005

Le Roux, F., Binesse, J., Saulnier, D., \& Mazel, D. (2007). Construction of a Vibrio splendidus mutant lacking the metalloprotease gene vsm by use of a novel counterselectable suicide vector. Applied and Environmental Microbiology, 73(3), 777-84. doi:10.1128/AEM.02147-06

Le Roux, F., Gay, M., Lambert, C., Waechter, M., Poubalanne, S., Chollet, B., Berthe, F. (2002). Comparative analysis of Vibrio splendidus -related strains isolated during Crassostrea gigas mortality events. Aquati Living Ressource, 15, 251-258.

Le Roux, F., Zouine, M., Chakroun, N., Binesse, J., Saulnier, D., Bouchier, C., Mazel, D. (2009). Genome sequence of Vibrio splendidus: an abundant planctonic marine species with a large genotypic diversity. Environmental Microbiology, 11(8), 1959-70. doi:10.1111/j.1462-2920.2009.01918.x

Le Roux, F. Le, Gay, M., Lambert, C., Nicolas, J. L., Gouy, M., \& Berthe, F. (2004). Phylogenetic study and identification of Vibrio splendidus-related strains based on gyrB gene sequences. Diseases of Aquatic Organisms, 58, 143-150.

Lindstedt, B.-A. (2005). Multiple-locus variable number 633 tandem repeats analysis for genetic fingerprinting of pathogenic bacteria. Electrophoresis, 26(13), $2567-2582$. doi:10.1002/elps.200500096

Lipp, ErinK. Rodriguez-Palacios, Cesar and Rose, J. (2001). Occurrence and distribution of the human pathogen Vibrio vulnificus in a subtropical Gulf of Mexico estuary. The Ecology and Etiology of Newly Emerging Marine Diseases, 159, 165-173.

Liu, R., Qiu, L., Yu, Z., Zi, J., Yue, F., Wang, L., ... Song, L. (2013). Identification and characterisation of pathogenic Vibrio splendidus from Yesso scallop (Patinopecten yessoensis) cultured in a low temperature environment. Journal of Invertebrate Pathology, 114(2), 144-50. doi:10.1016/j.jip.2013.07.005 
Lutthisungnoen, A., Kondo, S., Udomsopagit, S., Smittipat, N., \& Palittaponkarnpim, P. (2008). Characterization of Vibrio Cholerae O 1 Isolated During 2001-2004 by Variable Number of Tandem Repeat ( VNTR ) Typing, 274-281.

Macian, M., Ludwig, W., Aznar, R., Grimont, P., Schleifer, K., Garay, E., \& Pujalte, M. (2001). Vibrio lentus sp. nov., isolated from Mediterranean oysters. Int J Syst Evol Microbiol, 51(4), 1449-1456. doi:10.1099/00207713-51-4-1449

Maiden, M. C. J., Bygraves, J. A., Feil, E., Morelli, G., Russell, J. E., Urwin, R. Spratt, B. G. (1998). Multilocus sequence typing: A portable approach to the identification of clones within populations of pathogenic microorganisms. Proceedings of the National Academy of Sciences, 95(6), 3140-3145. doi:10.1073/pnas.95.6.3140

Mark Schena, Dari Shalon, Ronald W. Davis, 653 P. O. B. (1995). Quantitative Monitoring of Gene Expression Patterns with a Complementary DNA Microarray. Science, 270(5235), 467-470.

Mathur, J., Davis, B. M., \& Waldor, M. K. (2007). Antimicrobial peptides activate the Vibrio cholerae sigmaE regulon through an OmpU-dependent signalling pathway. Molecular Microbiology, 63(3), 848858. doi:10.1111/j.1365-2958.2006.05544.x

Mathur, J., \& Waldor, M. K. (2004). The Vibrio cholerae ToxR-regulated porin OmpU confers resistance to antimicrobial peptides. Infection and Immunity, 72(6), 3577-83. doi:10.1128/IAI.72.6.3577-3583.2004

Meistertzheim, A.-L., Arnaud-Haond, S., Boudry, P., \& Thébault, M.-T. (2013). Genetic structure of wild European populations of the invasive Pacific oyster Crassostrea gigas due to aquaculture practices. Marine Biology, 160(2), 453-463. doi:10.1007/s00227-012-2102-7

Nei, M., \& Li, W. H. (1979). Mathematical model for studying genetic variation in terms of restriction endonucleases. Proceedings of the National Academy of Sciences, 76(10), 5269-5273. doi:10.1073/pnas.76.10.5269

Nicolas, S.corre, G.Gauthier, R.Robert, D. A. (1996). bacterial problems associated with scallop Pecten maximus larval culture, 27(Standiford 1985), 67-76.

Olsen, J. S., Aarskaug, T., Skogan, G., Fykse, E. M., Ellingsen, A. B., \& Blatny, J. M. (2009). Evaluation of a highly discriminating multiplex multi-locus variable-number of tandem repeats (MLVA) analysis for 673 Vibrio cholerae. Journal of Microbiological Methods, 78(3), 271-85. doi:10.1016/j.mimet.2009.06.011

Pang, B., Yan, M., Cui, Z., Ye, X., Diao, B., Ren, Y., ... Kan, B. (2007). Genetic diversity of toxigenic and nontoxigenic Vibrio cholerae serogroups $\mathrm{O} 1$ and $\mathrm{O} 139$ revealed by array based comparative genomic hybridization. Journal of Bacteriology, 189(13), 4837-49. doi:10.1128/JB.01959-06

Paradis, E., Claude, J., \& Strimmer, K. (2004). APE: Analyses of Phylogenetics and Evolution in R language. Bioinformatics, 20(2), 289-290. doi:10.1093/bioinformatics/btg412

Saulnier D, Travers MA, D. D. S. (n.d.). Development of a duplex real-time PCR assay for rapid identification of Vibrio splendidus-related and $V$. aestuarianus strains from bacterial colony. See Related SOPs at Http://Www.eurl-mollusc.eu/SOPS.

Saulnier, D., De Decker, S., Haffner, P., Cobret, L., Robert, M., \& Garcia, C. (2010). A large scale epidemiological study to identify bacteria pathogenic to Pacific oyster Crassostrea gigas and correlation between virulence and metalloprotease-like activity. Microbial Ecology, 59(4), 787-98. doi:10.1007/s00248-009-9620-y

Sawabe, T., Kita-Tsukamoto, K., \& Thompson, F. L. (2007). Inferring the evolutionary history of vibrios by means of multilocus sequence analysis. Journal of Bacteriology, 189(21), 7932-6. doi:10.1128/JB.00693-07

Sawabe, T., Ogura, Y., Matsumura, Y., Feng, G., Amin, A. R., Mino, S., ... Hayashi, T. (2013). Updating the Vibrio clades defined by multilocus sequence phylogeny: proposal of eight new clades, and 694 the description of Vibrio tritonius sp. nov. Frontiers in Microbiology, 4(1-14). doi:10.3389/fmicb.2013.00414

Sébastien Lê, Josse Julie, F. H. (2008). FactoMineR : An R Package for Multivariate Analysis. Journal of Statistical Software, 25(1), 1-18.

Shannon, C. E. (1948). A Mathematical Theory of Communication. Reprinted with Corrections from The Bell System Technical Journal, 27, 379-423.

Shekar, M., Acharya, S., Karunasagar, I., \& Karunasagar, I. (2008). Variable repeat regions in the genome of Vibrio vulnificus and polymorphism in one of the loci in strains isolated from oysters. International Journal of Food Microbiology, 123(3), 240-5. doi:10.1016/j.ijfoodmicro.2008.02.008

Simpson, E. H. (1949). Measurement of diversity. Nature, 163, 688. doi:10.1038/163688a0

Stine, O. C., Alam, M., Tang, L., Sack, R. B., \& Morris, J. G. (2008). Seasonal Cholera from Multiple Small Outbreaks, Rural Bangladesh. Emerging Infectious Diseases, 14(5), 831-833. 
Tamura, K., Dudley, J., Nei, M., \& Kumar, S. (2007). MEGA4: Molecular Evolutionary Genetics Analysis (MEGA) software version 4.0. Molecular Biology and Evolution, 24(8), 1596-9. doi:10.1093/molbev/msm092

Teh, C. S. J., Chua, K. H., \& Thong, K. L. (2010). Multiple-locus variable-number tandem repeat analysis of Vibrio cholerae in comparison with pulsed field gel electrophoresis and virulotyping. Journal of Biomedicine \& Biotechnology, 2010, 817190. doi:10.1155/2010/817190

Thompson, F. L. (2003a). Vibrio fortis 715 sp. nov. and Vibrio hepatarius sp. nov., isolated from aquatic animals and the marine environment. International Journal of Systematic and Evolutionary Microbiology, 53(5), 1495-1501. doi:10.1099/ijs.0.02658-0

Thompson, F. L. (2003b). Vibrio kanaloae sp. nov., Vibrio pomeroyi sp. nov. and Vibrio chagasii sp. nov., from sea water and marine animals. International Journal Of Systematic And Evolutionary Microbiology, 53(3), 753-759. doi:10.1099/ijs.0.02490-0

Thompson, F. L., Thompson, C. C., \& Swings, J. (2003). Vibrio tasmaniensis sp. nov., isolated from Atlantic salmon (Salmo salar L.). Systematic and Applied Microbiology, 26(1), 65-9. doi:10.1078/072320203322337326

Thompson, J. D., Gibson, T. J., \& Higgins, D. G. (2002). Multiple sequence alignment using ClustalW and ClustalX. Current Protocols in Bioinformatics / Editoral Board, Andreas D. Baxevanis ... [et Al.], Chapter 2. doi:10.1002/0471250953.bi0203s00

Thompson, J. R., Pacocha, S., Pharino, C., Klepac-Ceraj, V., Hunt, D. E., Benoit, J., Polz, M. F. (2005). Genotypic diversity within a natural coastal bacterioplankton population. Science (New York, N.Y.), 307, 1311-1313. doi:10.1126/science.1106028

Urwin, R., \& Maiden, M. C. J. (2003). Multi-locus sequence typing: a tool for global epidemiology. Trends in Microbiology. doi:10.1016/j.tim.2003.08.006

Vanhove, A. S., Duperthuy, M., Charrière, G. M., Le Roux, F., Goudenège, D., Gourbal, B., Destoumieux-Garzón, D. (2014). Outer membrane vesicles are vehicles for the delivery of Vibrio tasmaniensis virulence factors to oyster immune cells. Environmental Microbiology, 32. doi:10.1111/1462-2920.12535

Vezzulli, L., Pezzati, E., Stauder, M., Stagnaro, L., Venier, 736 P., \& Pruzzo, C. (2014). Aquatic ecology of the oyster pathogens Vibrio splendidus and Vibrio aestuarianus. Environmental Microbiology. doi:10.1111/1462-2920.12484

Vora, G. J., Meador, C. E., Bird, M. M., Bopp, C. A., Andreadis, J. D., \& Stenger, D. A. (2005). Microarray-based detection of genetic heterogeneity, antimicrobial resistance, and the viable but nonculturable state in human pathogenic Vibrio spp. Proceedings of the National Academy of Sciences of the United States of America, 102(52), 19109-14. doi:10.1073/pnas.0505033102

Vos, P., Hogers, R., Bleeker, M., Reijans, M., van de Lee, T., Hornes, M., Kuiper, M. (1995). AFLP: a new technique for DNA fingerprinting. Nucleic Acids Research, 23(21), 4407-4414.

Waechter, M., Le Roux, F., Nicolas, J.-L., Marissal, É., \& Berthe, F. (2002). Caractérisation de bactéries pathogènes de naissain d'huître creuse Crassostrea gigas. Comptes Rendus Biologies, 325(3), 231-238. doi:10.1016/S1631-0691(02)01428-2

http://minisatellites.u-psud.fr

http://www.ebi.ac.uk/Tools/msa/clustalw2/

www.vibriology.net

\section{Tables}

Table 1. Reference strains used in this study.

Table 2

A. Primers and probe position designed in this study from the sequence of $V$. tasmaniensis (formerly $V$. splendidus- related) LGP32 genome. B. Validation of VNTR primers on V. splendidus clade and non clade Supplementary table S1. Distribution of strains isolated $(\mathrm{N}=435)$ by geographical origin and type of samples analyzed.

Supplementary table S2. Genbank accession numbers of ompU and vsm sequences from strains of the $V$. splendidus clade obtained in this study.

\section{Figures}

Fig.1. Distribution of strains isolated $(\mathrm{N}=435)$ by geographical origin and type of samples analyzed.

Fig.2. Representative agarose gel picture showing polymorphism for the VNTR region. Lane 1and 11: Smart Ladder (100 bp to $1000 \mathrm{bp}$ ), Lane 2: negative control, Lane 3: V. tasmaniensis (formerly $V$. 
splendidus- related) LGP32, Lanes 4 -10: $V$. splendidus clade isolates from the collection samples. Lane 4: strain 120 isolated from Spain (Sor River), Mytilus edulis in 2012; lane 5: strain 260 isolated from Spain (Sor River), Mytilus edulis in 2011; lane 6: strain 261 isolated from Spain (Sor River), Mytilus edulis in 2011; lane 7: strain 333 isolated from spain (Sor River), Mytilus edulis in 2011; lane 8: strain 271 isolated from Spain (Sor River), Crassostrea gigas in 2012; lane 9: strain 272 isolated from Spain (Sor River), Mytilus edulis in 2012; lane 10: strain 273 isolated from Spain (Sor River), Mytilus edulis in 2012.

Fig. 3. Two-dimensional hierarchical clustering of VNTR data of isolates of the $V$. splendidus clade based on presence/absence of distinct VNTR fragments. Fr: France, It: Italy, Sp: Spain, Ir: Ireland, Neth: Netherlands, C. gigas: Crassostrea 778 gigas, M. edulis: Mytilus edulis, M. Gallo: Mytilus galloprovincialis, +: isolation during oyster mortality peak, -: isolation outside oyster mortality peak, ND: mortality period not determined. High resolution image can be downloaded as Supplementary Figure 2.

Fig. 4. Phylogenetic tree of the gene encoding the outer membrane ompU on 102 European isolates of the $V$. splendidus clade. $V$. tasmaniensis LGP32 is underlined in red. The tree was built by the Neighbour-joining method based on sequence alignments using clustal W algorithm. Branch lengths are drawn to scale and proportional to the number of amino acid changes. Number at each node represents the percentage value given by bootstrap analysis of 1000 replicates.

Fig.5. Phylogenetic tree of the gene encoding the metalloprotease vsm on 97 European isolates of the $V$. splendidus clade. $V$. tasmaniensis LGP32 is underlined in red. The tree was built by the Neighbourjoining method based on sequence alignments using clustal $\mathrm{W}$ algorithm. Branch lengths are drawn to scale and proportional to the number of amino acid changes. Number at each node represents the percentage value given by bootstrap analysis of 1000 replicates.

Supplementary figure 1. Factor analysis of the VNTR data on 335 European V. splendidus isolates. Factor analysis was performed on the Burt table of VNTR-ompU- and vsm -PCR fragments obtained, and mortality data converted into binary data using code 1 or 0 according to presence or absence of each fragment. ND: not determined, It: Italy, Sp: Spain, Fr: France, Noth: Netherlands, Ir: Ireland, Dim 1 and 2: coordinates in the two most representative axis of the factor analysis A. Projection of strains coordinates using geographical origin and VNTR patterns (1 to 87) as supplementary variable in the analysis B. Projection of strains coordinates using sample type and VNTR pattern (1 to 87 ) as supplementary variable in the analysis. C. Projection of variables coordinates. Red dots: b1 to b16VNTR bands; vsm and ompU virulence factors both representing variables with two modes (P: present, A: absent) and mortality outbreaks representing variables with 3 modes. (P: present, $A$ : absent, NA: Not determined). Grey triangles: strains factorial coordinates are projected as above in S1A and S1B.

Fig. 1

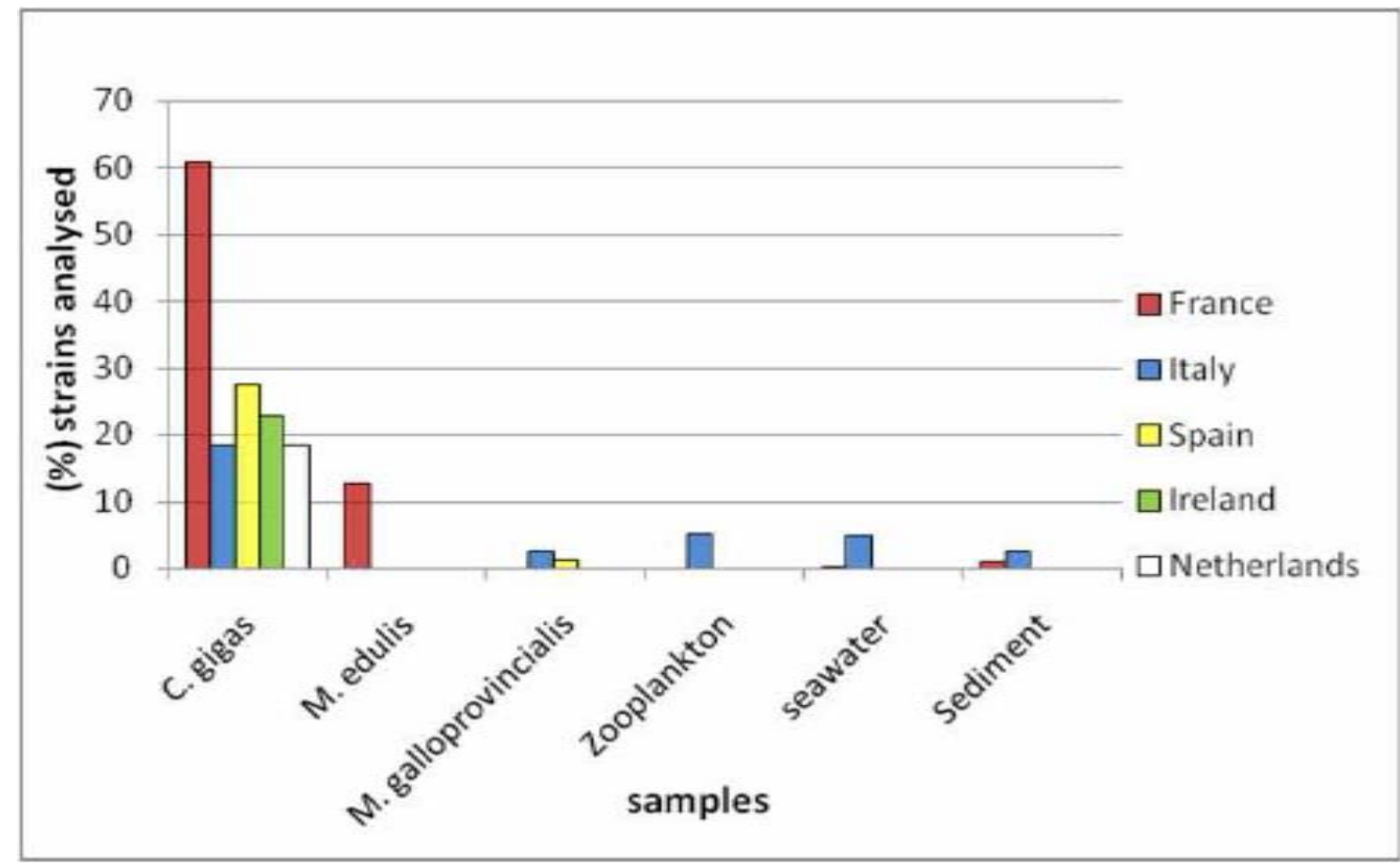


Fig. 2

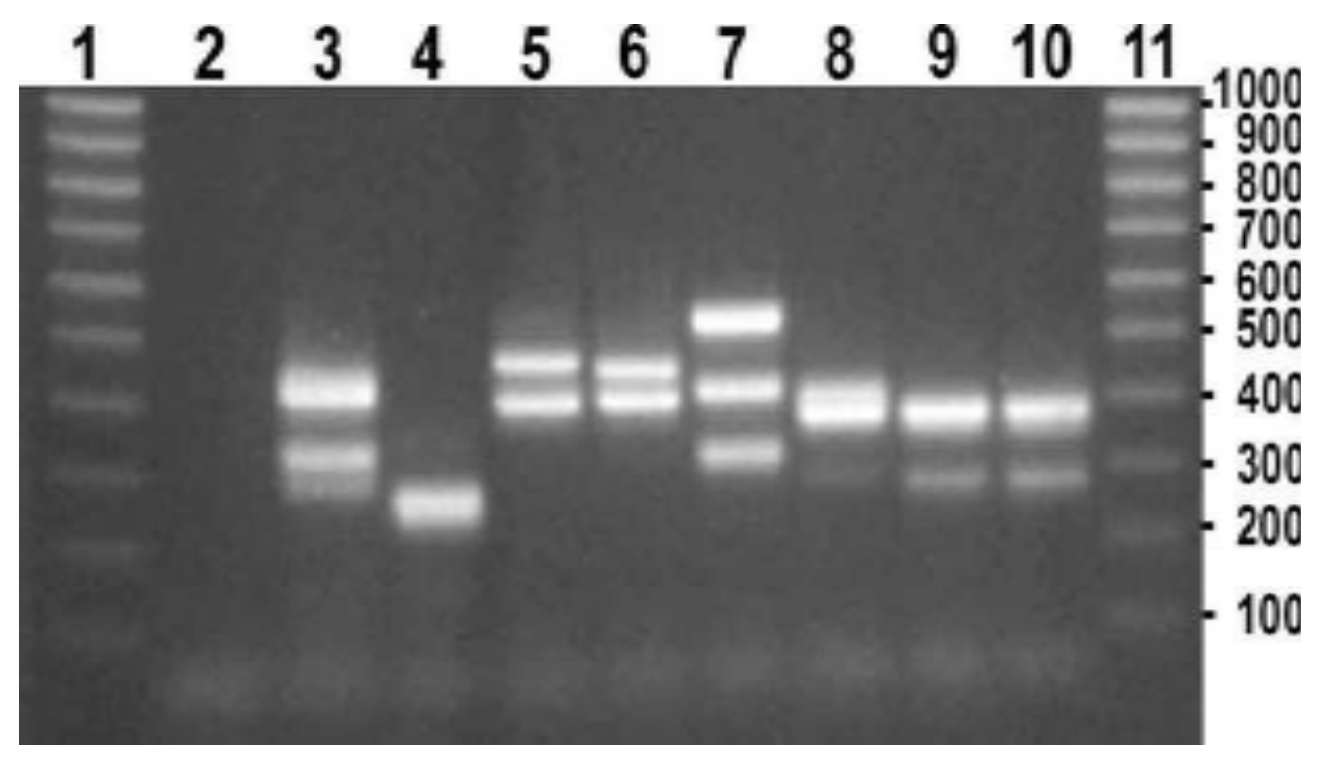




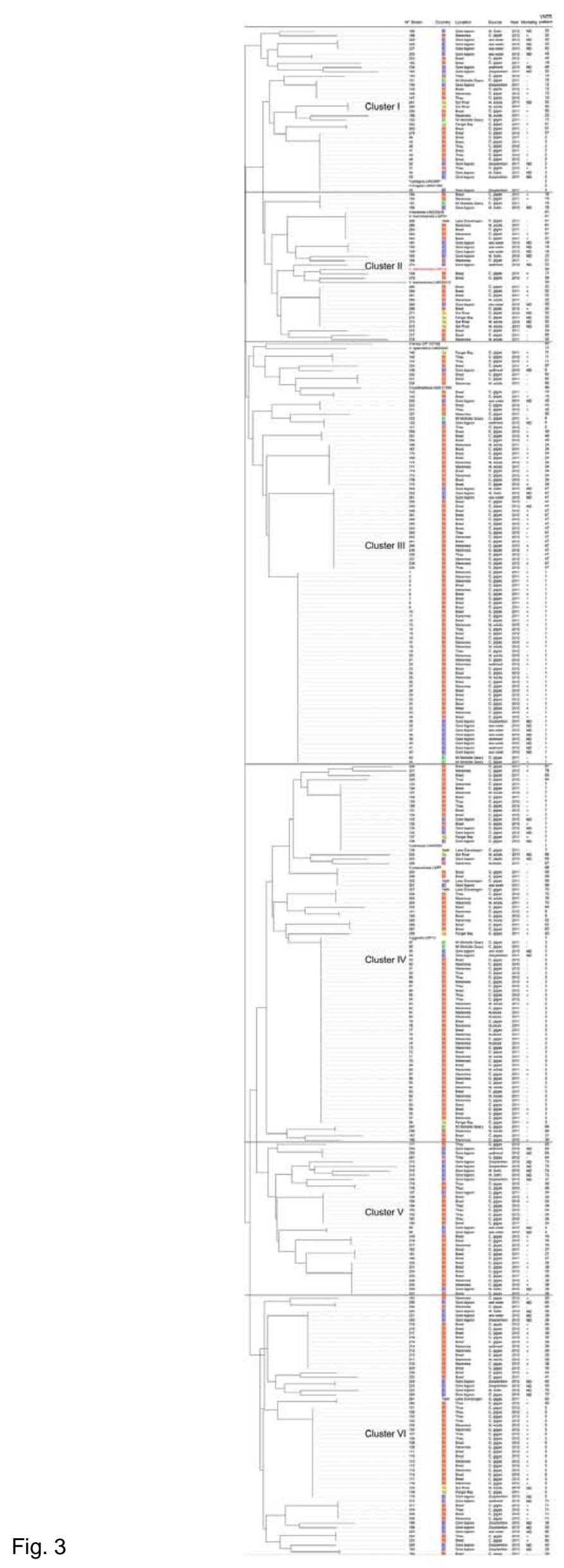




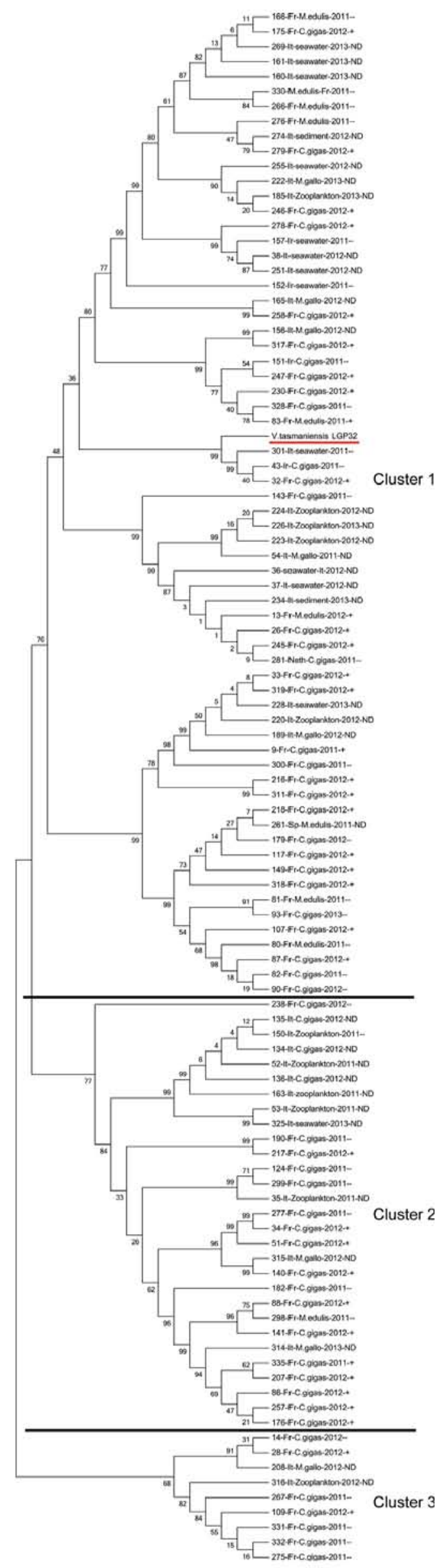

Fig. 4 
Fig. 5

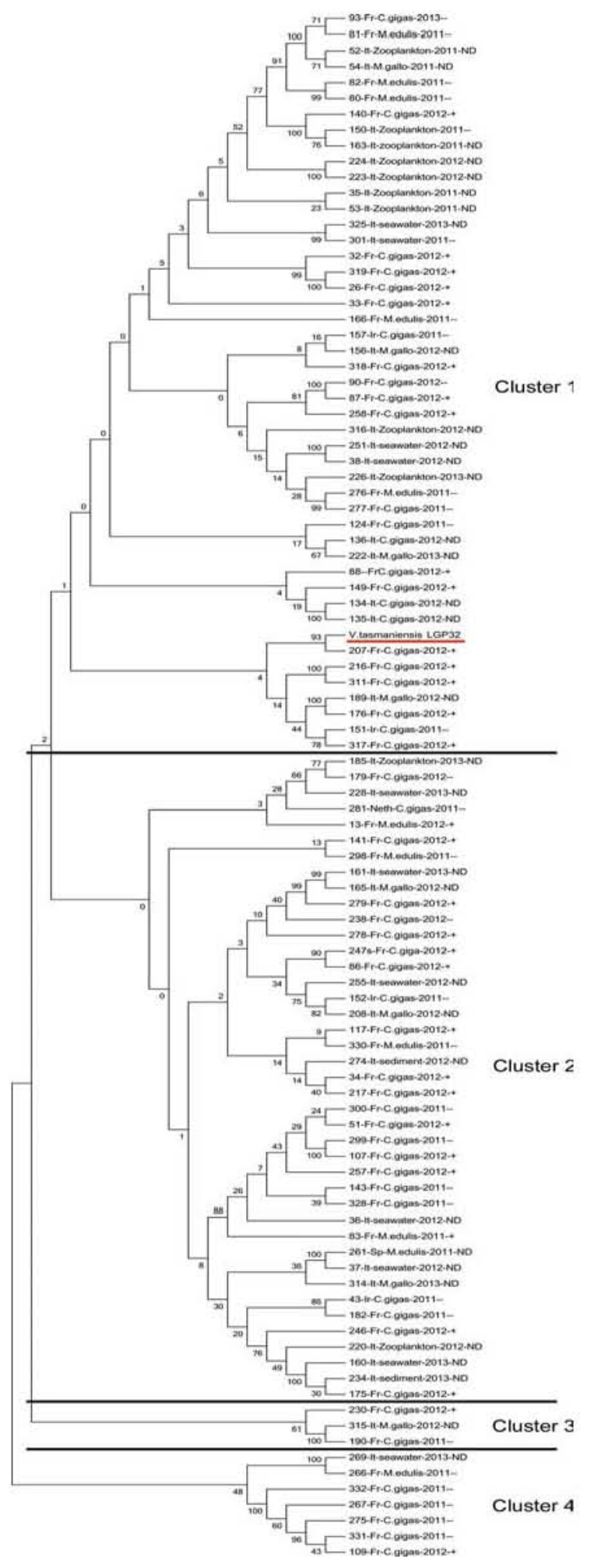


Tab. 1

\begin{tabular}{lll} 
Species & Strains & References \\
\hline V. gigantis & LGP 13 & Le Roux et al. (2005) \\
V. kanaloae & LMG20539 & Thompson (2003b) \\
V. tasmaniensis & LMG 20012 & Thompson et al. (2003) \\
V. lentus & CIP107166 & Macian et al. (2001) \\
V. chagasii & LMG 21353 & Thompson (2003b) \\
V. cyclitrophicus & LMG 21359 & Hedlund and Staley (2001) \\
V. harveyi & BB120 & Baumann et al. (1980) \\
V. crassostreae & LGP 7 & Faury et al. (2004) \\
V. Pelagius & LMG3897 & Baumann et al. (1971) \\
V. tapetis & CECT4600 & Borrego et al. (1996) \\
V. aestuarianus & O2/041 & Tiston and Seidler (1983) \\
V. splendidus & LMG 4042 & Baumann et al. (1980) \\
V. tasmaniensis & LGP 31 & Le Roux et al. (2009) \\
V. tasmaniensis & LGP 32 & Le Roux et al. (2009) \\
V. pomeroyi & LMG20537T & Thompson (2003b) \\
V. fortis & LMG20547T & Thompson (2003a) \\
\end{tabular}




\begin{tabular}{|c|c|c|c|}
\hline Character & Primer sequence (5'-3') & Position & Size (bp) \\
\hline Al & TGATGGCTGCGTATGATGG & 716891 & \multirow{2}{*}{416} \\
\hline A2 & GAAAAGCTCGTAGCGGCG & 717271 & \\
\hline B1 & GGTTCTCGAGCGTTAACGGT & 366603 & \multirow{2}{*}{321} \\
\hline B2 & GCGCTTGAAGGCTATAACGG & 366885 & \\
\hline $\mathrm{C} 1$ & TTACTTTACCGCGCTGTTAGG & 868940 & \multirow{2}{*}{337} \\
\hline $\mathrm{C} 2$ & CTCATCTAGATTCAGCTCATCGAC & 869233 & \\
\hline E1 & GCATACGGCCACTTCGG & 2844021 & \multirow{2}{*}{408} \\
\hline E2 & GGCAAAGAAGCATGAGTTACTG & 2844391 & \\
\hline F1 & TATTGAGCCTCCTATCATAAGAAC & 957312 & \multirow{2}{*}{315} \\
\hline F2 & CGTTACAGCCTGGTGAAAGC & 957584 & \\
\hline L1 & TCTGGTTCTCCTTCTGTTATGCC & 1290707 & \multirow{2}{*}{675} \\
\hline L2 & ССTAATCTATCTTCTCCTCGTGTGG & 1291335 & \\
\hline O1 & GAAGGTGTGATGCGTTTACTA & 2318164 & \multirow{2}{*}{281} \\
\hline $\mathrm{O} 2$ & GCCTCTCTTTTTGTTTTCTCG & 2318408 & \\
\hline R1 & TTGGTGCGAAAGAAGAGGTT & 1132493 & \multirow{2}{*}{325} \\
\hline R2 & CTGTTGAGATGACTCCATACGA & 1132777 & \\
\hline G1 & CCCAATGTTGGAAAACCAAAATC & - & - \\
\hline G2 & CTGAGAATGAAGTTGTATTAGAAGACT G & - & - \\
\hline H1 & CGAACAAAGCCCGCACC & - & - \\
\hline $\mathrm{H} 2$ & GAGGATGACGAATGACAAGCC & - & - \\
\hline D1 & GCTATGATTCACGGCTTTGC & - & - \\
\hline D2 & ACTGACTTTGCTGGCGGC & - & - \\
\hline $\mathrm{J} 1$ & CCCTGTGAGCCGTGGTTG & - & - \\
\hline $\mathrm{J} 2$ & ATTGGTTGCGGAAGGCG & - & - \\
\hline K1 & TTGAACAAACCAAAGAAGCCAG & - & - \\
\hline $\mathrm{K} 2$ & GATGACGGAAAAAAAGAAGCG & - & - \\
\hline M1 & CCAACTATCACAAACAGCCCTTA & - & - \\
\hline M2 & CTAAGACAGACAGTGTTGCTAACCG & - & - \\
\hline N1 & GGTTTGTTAGATGAAGCGAAGG & - & - \\
\hline $\mathrm{N} 2$ & GCGTGCTCTGTTTGGTGATA & - & - \\
\hline 01 & GAAGGTGTGATGCGTTTACTA & - & - \\
\hline $\mathrm{O} 2$ & GCCTCTCTTTTTGTTTTCTCG & - & - \\
\hline P1 & GTCAGGTAGAAAAGGTGGTCAG & - & - \\
\hline P2 & CGTGCCCTCTCAAGTCAATG & - & - \\
\hline Q1 & GTTTATCGGCTTGGGGAGTA & - & - \\
\hline Q2 & CAGCAAAGTCACGGCAAAAT & - & - \\
\hline S1 & TTGGTTAGGTGGTGGTGTGA & - & - \\
\hline S2 & CTTGGGTAGGTGCGGTGAT & - & - \\
\hline $\mathrm{T} 1$ & TTGACCCTCTACTCCACGATG & - & - \\
\hline $\mathrm{T} 2$ & GTGCGGCGCTTTTATCTG & - & \multirow{4}{*}{153} \\
\hline $16 \mathrm{~S} \mathrm{SpF} 1$ & ATCATGGCTCAGATTGAACG & 28 & \\
\hline $16 \mathrm{~S} \mathrm{SpR1}$ & CAATGGTTATCCCCCACATC & \multirow[t]{2}{*}{161} & \\
\hline $16 \mathrm{~S}$ probe & FAM-CCCATTAACGCACCCGAAGGATTG-BHQ1 & & \\
\hline OmpU F & GTCCTACAGACAGCGATAGC & \multirow{2}{*}{ VS-2494 } & \multirow[t]{2}{*}{769} \\
\hline OmpU R & GTGGTAAGCCATGATATCGG & & \\
\hline Vsm F & TACAAAGAKTGGATGAACAC & \multirow[t]{2}{*}{ VS-1267 } & \multirow[t]{2}{*}{531} \\
\hline Vsm R & TACTGTTTGCCGTCCAATAC & & \\
\hline
\end{tabular}


$V$. gigantis LGP 13

$-\ldots+-\ldots+\cdots$

V. crassostreae LGP 7

V.kanaloae LMG20539

V. lentus CIP107166

V. chagasii LMG 21353
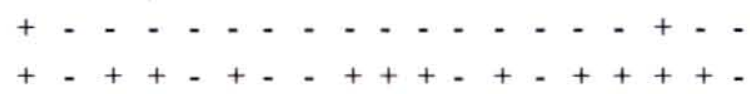

V. cyclotrophicus LMG 21359

V. pelagius LMG3897

V. splendidus LMG 4042

V. tasmaniensis LMG 20012

V. tasmaniensis LGP 32

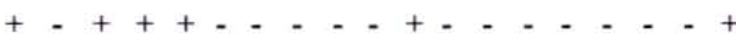

V. tasmaniensis LGP 31

V. pomeroyi LMG20537T

$V$. fortis LMG20547T

V. harveyi $\mathrm{BB} 120$

V. tapetis CECT4600

V. aestuarianus $02 / 041$

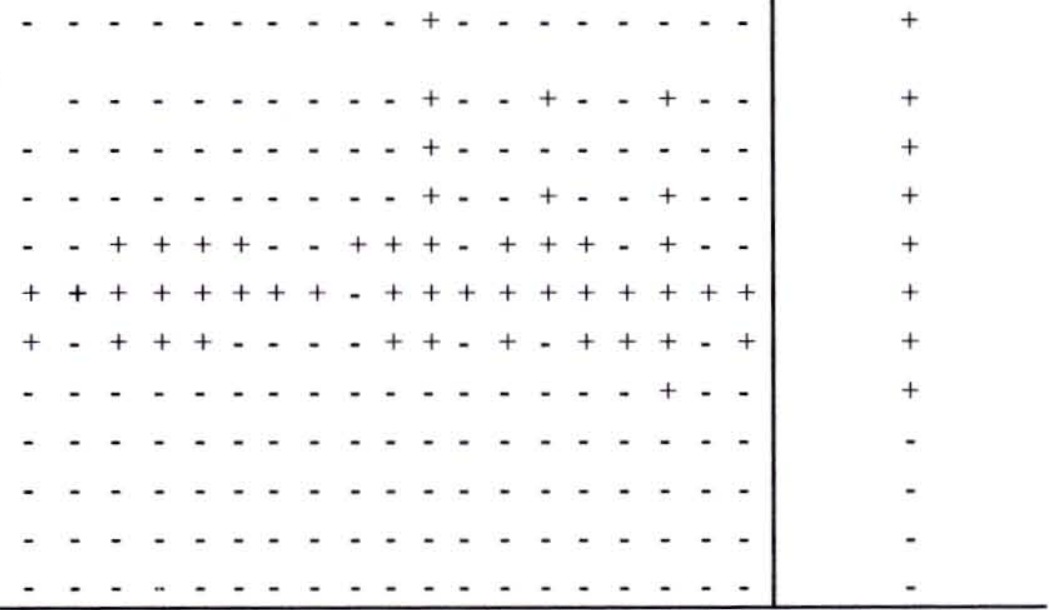

